\title{
Malignant peripheral nerve sheath tumor staging on pet-ct Imaging in a patient with neurofibromatosis type I
}

\begin{abstract}
Malignant peripheral nerve sheath tumors can be seen in about $4.6 \%$ of neurofibromatosis type I patients. In this rare case, we aimed to emphasize the importance of PET-CT imaging in demonstrating the prevalence of a 24year-old male patient with neurofibromatosis type 1 .
\end{abstract}

Keywords: neurofibromatosis, acquisition mode, lymphadenopathies, necrotic hypermetabolic, left pararectal, hypermetabolic, right hemithorax
Volume 3 Issue 4 - 2017

\author{
Pelin Ozcan Kara,' Zehra Pinar Koc,' Emel \\ Sezer, ${ }^{2}$ Kadir Eser, ${ }^{2}$ Vehbi Ercolak ${ }^{2}$ \\ 'Department of Nuclear Medicine, Mersin University, Turkey \\ ${ }^{2}$ Department of Oncology, Mersin University, Turkey
}

\begin{abstract}
Correspondence: Pelin Ozcan Kara, Mersin University Hospital, Department of Nuclear Medicine, 33343 Mersin, Turkey, Tel 9032424I0000, Fax 9032424I0098, Email ppelinozcan@gmail.com
\end{abstract}

Received: July 01, 2017 | Published: July 17, 2017
Abbreviations: MPNST, malignant peripheral nerve sheath tumor; FDG, fludeoxyglucose; PET-CT, positron emission tomography-computed tomography; NF1, neurofibromatosis-1

\section{Introduction}

Malignant peripheral nerve sheath tumor (MPNST) is a rare tumor group seen in neurofibromatosis type I patients. There are few reports on PET-CT imaging of this rare entity in literature. FDG PET$\mathrm{CT}$ is a part of the conventional work-up among a variety of cancer patients. Nevertheless, in certain clinical situations PET-CT may be of great significance, especially as treatment algorithm or differential diagnosis of benign and malign tumors. In this rare case, we aimed to emphasize the importance of PET-CT imaging in demonstrating the prevalence of a 24year-old male patient with neurofibromatosis type 1 .

\section{Case report}

A 24year-old male patient followed up neurofibromatosis type I in bilateral lower extremity was sent to our Nuclear Medicine Department for PET-CT imaging for suspected lung metastasis on radiological imaging. Following 8 hours of fasting, while the patient had a blood glucose level of $79 \mathrm{mg} / \mathrm{dL}, 8.7 \mathrm{mCi}(321.7 \mathrm{MBq})$ 18F-FDG i.v. was injected. PET-CT scan was obtained $60 \mathrm{~min}$ after injection using an integrated scanner (GE Discovery PET-CT 610, US). A whole-body CT scan was performed without intravenous contrast administration with $130 \mathrm{kV}, 50 \mathrm{mAs}$, a pitch of 1.5 , a section thickness of $5 \mathrm{~mm}$, and a field of view of $70 \mathrm{~cm}$. A PET scan was performed immediately after an unenhanced CT scan, and acquired from the calvarium to the footwell with a 2.3 -min acquisition per bed position using a threedimensional acquisition mode. Obtained images were evaluated after attenuation correction with low dose nondiagnostic CT. PETCT imaging demonstrated hypermetabolic (SUVmax: 5.23-10.08) pleural thickening and effusion on the right hemithorax extending to perihepatic region and diaphragmatic crus and several hypermetabolic metastatic nodules with the largest size approximately $3 \mathrm{~cm}$ (SUVmax: 15.46) in the basal segment of the left lower lobe of the lung. Bilateral inguinal lymphadenopathies and necrotic hypermetabolic masses (SUVmax: 5.05) with a largest diameter of approximately 9x6 $\mathrm{cm}$ were observed on the right and left pararectal areas in pelvic region. In addition, lymphedeme in both lower extremities, neurofibromatosis masses in the right gluteal region and in soft tissues at different levels in both lower extremities with similar characteristics (SUVmax: 6.48) as pelvic masses were observed (Figure 1).

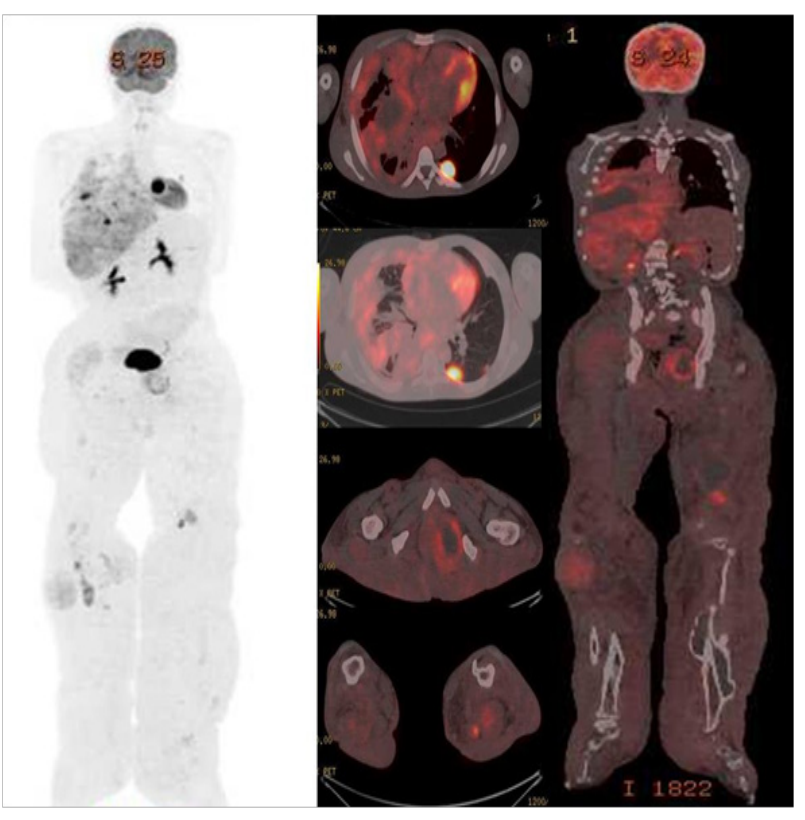

Figure I PET/CT MIP (Maximum intensity projection) and axial fusion images demonstrates symmetrical diffuse markedly increased metabolic activity including all the cross-sectional area in skeletal muscle structure.

\section{Discussion}

Malignant peripheral nerve sheath tumor (MPNST) constitutes the tumor group, also called neurogenic sarcoma, neurofibrosarcoma, malign neurilemmom, malign schwannoma. Tumors seen in these 
group of patients are at worse prognosis due to early onset, central localization, large size, more frequent metastases and recurrences. There are few reports on this rare entity in literature. ${ }^{1-7}$ In a case report, the authors present a typical case of MPNST in a 36-year-old man with NF type 1, which occurred on the left buttock. A PET-CT showed findings of possible inguinal lymph node metastasis and a lymph node biopsy confirmed the diagnosis. ${ }^{1}$ In an other report the authors presented a clinical case with Von Recklinghausen disease and recurrence of a left dorsal neurofibrosarcoma diagnosed by Positron Emission Tomography with fluorine-18-fluoro- exyD-glucose. ${ }^{2}$ In rare cases, PET-CT has been helpful and guiding in the imaging of the disease and in the staging of the disease. The authors reported the case of a patient with NF1 who developed synchronous malignant peripheral nerve sheath tumors, one with a focus of angiosarcoma. ${ }^{3}$ In a very recent original article on determining whether measurements of ${ }^{18} \mathrm{~F}-\mathrm{FDG}$ heterogeneity could improve differentiation of benign symptomatic neurofibromas from malignant peripheral nerve sheath tumours (MPNSTs), ${ }^{8}$ F-FDG PET data from a cohort of 54 patients with neurofibromatosis-1 (NF1), and clinically suspected malignant transformation of neurofibromas into MPNSTs, were included. The authors concluded that ${ }^{8} \mathrm{~F}-\mathrm{FDG}$ uptake in MPNSTs is higher than benign symptomatic neurofibromas, as defined by SUV parameters, and more heterogeneous, as defined by first- and highorder heterogeneity parameters. ${ }^{4}$ We believe the current interesting case is important due to the relatively uncommon clinical presentation and the discussion on the utility of 18 -FDG PET-CT in this very rare entity.

\section{Acknowledgements}

None.

\section{Conflict of interest}

Author declares that there is no conflict of interest.

\section{References}

1. Kang MJ, Kang H, Kim HO, et al. A Case of Malignant Peripheral Sheath Tumor Arising from Neurofibromatosis Type 1. Ann Dermatol. 2008;20(1):32-36.

2. Ruíz-Hernández G, Hornedo-Muguiro J, Salinas-Hernández P, et al. Positron emission tomography in a patient with Von Recklinghausen disease and left dorsal neurofibrosarcoma. Rev Esp Med Nucl. 2003;22(6):418-423.

3. Winters AC, Black JO, Cost CR. Metastatic angiosarcoma arising in malignant peripheral nerve sheath tumor in a young patient with neurofibromatosis type 1. Pediatr Blood Cancer. 2017:64(12).

4. Cook GJR, Lovat E, Siddique M, et al. Characterisation of malignan peripheral nerve sheath tumours in neurofibromatosis-1 using heterogeneity analysis of 18F-FDG PET. Eur J Nucl Med Mol Imaging. 2017;44(11):1845-1852.

5. Van Der Gucht A, Zehou O, Djelbani-Ahmed S, et al. Metabolic Tumour Burden Measured by 18F-FDG PET/CT Predicts Malignant Transformation in Patients with Neurofibromatosis Type-1. Plos One. 2016;11(3):e0151809.

6. Lieber B, Han B, Allen J, et al. Utility of positron emission tomography in schwannomatosis. Journal of Clinical Neuroscience. 2016;30:138-140.

7. Salamon J, Papp L, Tóth Z, et al. Nerve Sheath Tumors in Neurofibromatosis Type 1: Assessment of Whole-Body Metabolic Tumor Burden Using F-18-FDG PET/CT. Plos One. 2015;10(12):e0143305. 